\title{
The Simon effect based on the egocentric and allocentric reference frame
}

\author{
Huan Wang ${ }^{1}$ Nan Liu ${ }^{1}$ Guiying Zou ${ }^{1} \cdot \mathrm{Hui} \mathrm{Li}^{1} \cdot$ Hang Zeng ${ }^{1}$. \\ Jiedan Chen ${ }^{1} \cdot$ Qi Chen ${ }^{1,2}$
}

Published online: 24 November 2015

(C) The Psychonomic Society, Inc. 2015

\begin{abstract}
The classic Simon effect refers to the phenomenon that responses are faster when the task-irrelevant egocentric stimulus location is on the same side as the response hand than when not. However, the spatial location of an object often varies according to which reference frame the object location was represented in, e.g., egocentric versus allocentric reference frame. It has been unknown, however, whether and how the Simon effect based on the egocentric and the allocentric reference interact, and whether the potential interaction between them is affected by task demands. To investigate these questions, we orthogonally crossed the stimulus-response compatibility induced by the egocentric and the allocentric positions during the Simon effect. Moreover, the irrelevant egocentric and allocentric positions were either explicitly represented via interleaving spatial and non-spatial tasks in Experiment 1 or not in Experiment 2. The results suggested that the Simon effect based on the egocentric locations occurred irrespective of whether the egocentric representations were explicitly coded based on current task demands or not. The Simon effect based on allocentric locations, however, seemed to occur only when the allocentric representations were explicitly induced. Moreover, the egocentric and allocentric Simon effect interacted in a similar way irrespective of task demands, with significantly delayed
\end{abstract}

Qi Chen

qi.chen27@gmail.com

1 Center for Studies of Psychological Application and School of Psychology, South China Normal University, Guangzhou 510631, China

2 Epilepsy Center, Guangdong 999 Brain Hospital, Guangzhou 510631, China responses when both the egocentric and the allocentric locations were incongruent with the response code.

Keywords Allocentric reference frame $\cdot$ Egocentric reference frame $\cdot$ Simon effect

\section{Introduction}

Stimulus response compatibility (SRC) refers to the phenomenon that participants usually respond more quickly and with fewer errors when the spatial positions of the target and the response hand are congruent than when they are incongruent (Fitts \& Deininger, 1954; Fitts \& Seeger, 1953). For example, the Simon effect is a typical kind of SRC (Simon \& Small, 1969). In the classic Simon effect paradigm, participants are asked to discriminate a physical feature (e.g., color) of an object which appears randomly on the left or right side of the mid-sagittal line of the screen by pressing a left key if the target is of one color and a right key if the target is of another color. Although the target location is irrelevant to the color discrimination task, participants' performance turns out to be better when the task-irrelevant location is congruent with the side of the response key than when incongruent (Hedge \& Marsh, 1975; Hommel, 1994; Kornblum, Hasbroucq, \& Osman, 1990; Lu \& Proctor, 1995; Marble \& Proctor, 2000; Simon, 1990; Simon \& Small, 1969). The Simon effect exists in spite of a variety of experimental manipulations, such as the relevant stimulus dimension, type of stimulus, presentation, and reaction mode etc. (Hedge \& Marsh, 1975; Hommel, 1994; Kornblum et al., 1990; Lamberts, Tavernier, \& d'Ydewalle, 1992; Proctor \& Pick, 2003; Simon, 1990; Umiltà \& Liotti, 1987; Vallesi, Mapelli, Schiff, Amodio, \& Umilta, 2005; Wühr, 2006). 
In the classic Simon effect, the manipulation of stimulus-response compatibility is always between the egocentric position of the target and the side of the response key. In the real world, however, object positions can be coded either in the egocentric (relative to the mid-sagittal line of the observer's body or body parts) or the allocentric (relative to another object or a background independent of the observer) reference frame (Bisiach, Capitani, \& Porta, 1985; Driver \& Halligan, 1991; Marshall \& Halligan, 1993; Walker, 1995). Therefore, upon the appearance of an object, its egocentric position (left vs. right side of the mid-sagittal line) could be either congruent or incongruent with the side of the response key, and similarly its allocentric position relative to another background object could be either congruent or incongruent with the side of the response key as well. By orthogonally combining the allocentric and egocentric Simon effect congruency in the present study, we aimed to investigate whether the Simon effect existed based on both the allocentric and egocentric positions and whether there was a potential interaction between them.

In addition, it has been suggested that participants tend to use the egocentric reference frame when they were not explicitly instructed which specific frame to adopt. For instance, when participants were asked to judge the position of one of the two simultaneously presented objects, more participants adopted the egocentric reference frame to localize the objects than those who used the allocentric reference frame (Tversky $\&$ Hard, 2009). These results implied that the egocentric reference frame may be more salient, and thus has primacy over the allocentric reference frame. In the real world, people explore the external environment through their own eyes and body parts. Therefore, egocentric representations are generated automatically, whereas encoding allocentric representations would demand extra cognitive resources (Epley, Morewedge, \& Keysar, 2004). We thus hypothesized that the coding of egocentric and allocentric representations may be differentially affected by task demands: the egocentric representations might be automatically coded regardless of the task demand, whereas the allocentric representations may require an explicit spatial coding task demand to exert an effect. Therefore, we further aimed to investigate how task demands affect the Simon effects based on the egocentric and allocentric reference frames and the potential interaction between them. Specifically, in Experiment 1, we alternated the egocentric and the allocentric spatial judgment tasks with the color discrimination task, to make sure that both the egocentric and the allocentric object positions were explicitly induced and coded based on the current task demands of allocentric and egocentric judgments. We predicted that in Experiment 1 the allocentric and egocentric representations would be explicitly induced through corresponding spatial judgment tasks, and accordingly the two types of representations would interfere with the response codes, inducing the Simon effects based on both frames of reference. By contrast, in Experiment 2 only the color discrimination task, rather than the spatial judgment tasks, was performed on the same stimulus set as that in Experiment 1, so that it was not necessary for any spatial representations to be explicitly coded. We accordingly predicted that a significant Simon effect may occur based only on the egocentric, rather than the allocentric, reference frame in Experiment 2.

\section{Experiment 1}

\section{Method}

\section{Participants}

Twenty healthy participants (12 female and eight male, 1824 years old) took part in the present experiment. They were all right-handed, and had normal or corrected-to-normal vision as well as normal color vision. All the participants gave informed consent in accordance with the Declaration of Helsinki prior to the experiment and got paid for their participation afterwards. The study was approved by the Ethics Committee of School of Psychology, South China Normal University.

\section{Apparatus, stimuli, and experimental setup}

The experiment was run in a dimly lighted room. All the visual stimuli were presented on a computer screen with a distance of $59 \mathrm{~cm}$ to participants' eyes. The stimuli in each trial consisted of two objects: a fork on the top of a round orange plate (Fig. 1). The fork was presented as either light or dark gray (values of RGB (red, green, blue): 236, 236, 236, and $68,68,68$, respectively). The diameter of the plate was $15.0^{\circ}$ visual angle, and the nearest end of the fork was $2.5^{\circ}$ visual angle. The egocentric locations of the fork (with respect to the mid-sagittal plane of the participants' body), and the allocentric locations of the fork (with respect to the mid-sagittal plane of the plate) were orthogonally varied. The fork could be located at four different locations with respect to the mid-sagittal plane of the participants' body, i.e., $-4.7^{\circ},-3.1^{\circ}, 3.1^{\circ}$, and $4.7^{\circ}$, forming four different egocentric positions of the fork. Meanwhile, the fork could intersect the plate at four different locations with regard to the mid-sagittal plane of the plate, i.e., $-4.9^{\circ},-3.0^{\circ}, 3.0^{\circ}$, and $4.9^{\circ}$, forming four different allocentric positions of the fork (Fig. 1). The allocentric and egocentric positions of the fork were derived from a psychophysical test prior to the formal experiment, in which we adjusted the visual angles of the allocentric and egocentric positions of the targets so that task difficulty of allocentric and egocentric judgments was comparative. 


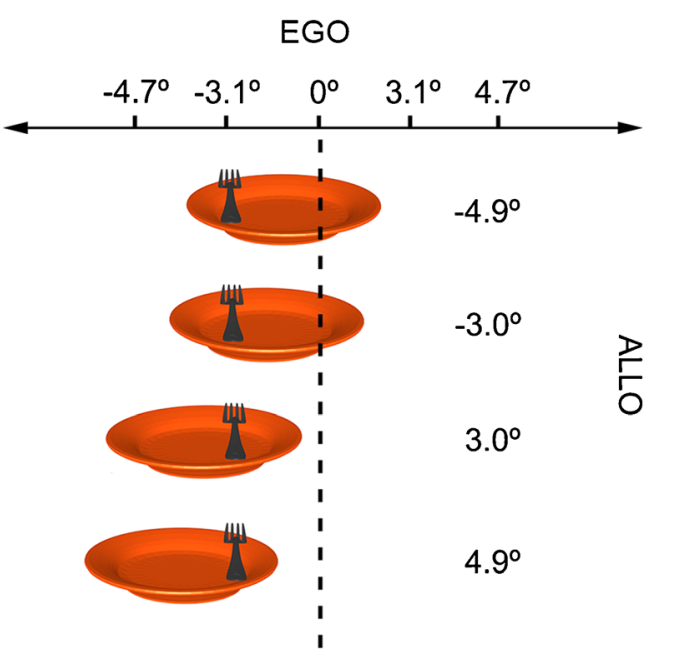

Fig. 1 Experimental stimuli. The stimuli consisted of a fork intersecting an orange plate. The fork was presented either in light gray or in dark gray. In the spatial judgment tasks, participants were asked to judge the position of the fork with reference either to the plate or to their midsagittal plane, and in the color discrimination task, they were asked to judge the color of the fork. There were four egocentric positions for the fork. For each of the four egocentric locations of the fork $\left(-4.7^{\circ},-3.1^{\circ}\right.$, $3.1^{\circ}$, and $4.7^{\circ}$ ) the location of the plate was varied independently around the fork, forming four possible allocentric positions $\left(-4.9^{\circ},-3.0^{\circ}, 3.0^{\circ}\right.$, and $\left.4.9^{\circ}\right)$. The black dotted line indicates the invisible mid-sagittal line

\section{Experimental design and tasks}

There were three types of tasks: egocentric task, allocentric task, and color discrimination task. In the egocentric judgment task, participants were asked to judge whether the fork was on the left or right side of the mid-sagittal plane of their own body. In the allocentric judgment task, participants were asked to judge whether the fork was on the left or right side of the plate. For both spatial tasks, participants were instructed to press the left or right button on the response pad with the index finger of their left or right hand corresponding to the left or right side judgment, respectively. In the color discrimination task, participants were asked to judge whether the color of the fork was light or dark by pressing the left or right button on the response pad with the index finger of their left or right hand, respectively. The correspondence between the two colors and the response buttons was counter-balanced across participants. The three types of tasks were blocked and alternated with each other. Every egocentric or allocentric judgment block was followed by a color discrimination block. Each block began with a 3-s visual instruction, informing the participants of the type of task in the upcoming block. Within each block, all types of trials were randomly mixed. To force the participants to use the mid-sagittal plane of their own body, rather than the central fixation, as a reference in the egocentric spatial task, we chose not to use a central fixation. Furthermore, participants were required to keep their gaze straightforward without moving their eyes throughout the experiment. The target was presented for a short duration of $250 \mathrm{~ms}$ in each trial to prevent eye movements. The duration of each trial was $2,000-3$, $000 \mathrm{~ms}$ to avoid potential temporal expectations. Eight trials were presented per block, resulting in a block duration of 20-30 s. There were 12 repetitions for each of the two types of spatial judgment blocks, and 24 repetitions for the color discrimination block. All the participants completed a training session of $3 \mathrm{~min}$ before the formal experiment started. The three types of tasks were alternatively performed in both the practice and the formal experiments.

For spatial judgment tasks, the egocentric and allocentric positions of the target could be either congruent or incongruent. Thereby, based on the spatial congruency between the egocentric and allocentric positions, the stimulus could be classified into two types. Thus, the present experimental design in the spatial judgment tasks was a 2 (type of task: egocentric vs. allocentric) $\times 2$ (the congruency between egocentric and allocentric positions: congruent vs. incongruent) two-factorial within-subject design. There were four experimental conditions altogether, and 48 trials in each experimental condition.

For the color discrimination task, critically, two types of spatial congruency were manipulated: the congruency between the egocentric position of the target and the side of response, and the congruency between the allocentric position of the target and the side of response. The two types of spatial congruency were orthogonally crossed so that the experimental design in the color discrimination task was a 2 (the egocentric congruency: congruent vs. incongruent) $\times 2$ (the allocentric congruency: congruent vs. incongruent) two-factorial within-subject design. There were four experimental conditions in total, and 48 trials in each experimental condition.

\section{Results}

Incorrect responses and reaction times (RTs) exceeding 3 SDs larger/smaller than the mean RT in each condition were excluded from further analysis. Three participants' data were removed as their error rates were over $5 \%$.

\section{Spatial judgment tasks}

In spatial tasks no significant difference between egocentric and allocentric tasks was found for RTs, $t_{(16)}=0.15, p=0.88$, indicating that RTs in the egocentric judgment $(627 \mathrm{~ms})$ and allocentric judgment $(625 \mathrm{~ms})$ were comparable. For error rates, similar to the results for RTs, no significant difference between egocentric and allocentric tasks was obtained, $t_{(16)}=1.01, p=0.33$, indicating that error rates in the egocentric judgment $(2.5 \%)$ and allocentric judgment $(1.8 \%)$ were comparable. 


\section{Color discrimination task}

For the color discrimination task, mean RTs and error rates (\%) in each experimental condition were submitted to a 2 (the egocentric congruency: congruent vs. incongruent) $\times 2$ (the allocentric congruency: congruent vs. incongruent) repeated-measures ANOVA.

For RTs, the main effect of the egocentric congruency was significant, $F_{(1,16)}=21.34, p<0.01$. The RTs in the egocentric incongruent condition (647 ms) were significantly slower than the RTs in the egocentric congruent condition (597 ms), i.e., the classical Simon effect based on the egocentric positions. The main effect of the allocentric congruency was significant as well, $F_{(1,16)}=25.03, p<0.01$, indicating that the RTs in the allocentric incongruent condition (643 ms) were significantly slower than the RTs in the allocentric congruent condition (601 ms), i.e., the Simon effect based on the allocentric positions. The two-way interaction was also significant, $F_{(1,16)}=16.94, p<0.01$ (Fig. 2a). Further tests on simple effects were performed to clarify the interaction. For the egocentric congruent condition, RTs in the allocentric incongruent condition (605 ms) were marginally longer than RTs in the allocentric congruent condition (589 ms), $t_{(16)}=$ $1.93, p=0.07$. For the egocentric incongruent condition, RTs in the allocentric incongruent condition $(681 \mathrm{~ms})$ were significantly longer than RTs in the allocentric congruent condition (613 ms), $t_{(16)}=5.49, p<0.01$. For the allocentric congruent
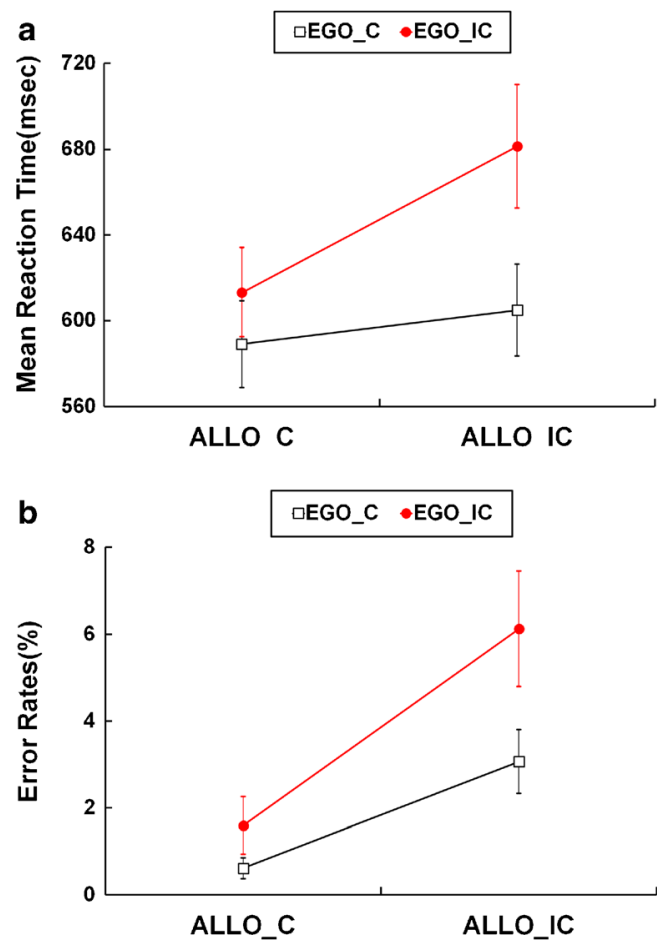

Fig. 2 Mean reaction times (a) and error rates (b) in the four experimental conditions of the color discrimination task in Experiment 1. The error bars represent standard errors condition, RTs were significantly longer in the egocentric incongruent condition (613 ms) than in the egocentric congruent condition $(589 \mathrm{~ms}), t_{(16)}=2.64, p=0.02$. For the allocentric incongruent condition, RTs were significantly longer in the egocentric incongruent condition $(681 \mathrm{~ms})$ than in the egocentric congruent condition (605 ms), $t_{(16)}=4.99, p<0.01$. Therefore, RTs were the longest when both the allocentric and the egocentric locations were incongruent with the response hand, and were the shortest when both the allocentric and the egocentric locations were congruent with the response hand. Moreover, the significant two-way interaction suggested that the Simon effect based on the egocentric reference frame was significantly larger in the allocentric incongruent condition $(77 \mathrm{~ms})$ than in the allocentric congruent condition (24 ms). Similarly, the Simon effect based on the allocentric reference frame was significantly larger in the egocentric incongruent condition $(68 \mathrm{~ms})$ than in the egocentric congruent condition (16 ms).

For error rates, the main effect of the egocentric congruency was marginally significant, $1.8 \%$ versus $3.9 \%, F_{(1,16)}=$ $4.02, p=0.06$, showing a trend of significant Simon effect based on the egocentric reference frame. The main effect of the allocentric congruency was significant as well, $1.1 \%$ versus $4.6 \%, F_{(1,16)}=26.39, p<0.01$. The two-way interaction was not significant, $F_{(1,16)}=2.67, p=0.12$, but the pattern of error rates was similar to that of the RTs (Fig. 2b).

To further examine whether the allocentric versus egocentric judgment tasks could effectively induce the coding of the corresponding spatial representations and whether the different types of the explicitly coded spatial representations could affect the sizes of the different types of Simon effects in the following color discrimination tasks, we submitted the sizes of the different types of Simon effects (i.e., incongruent RT > congruent RT) to a 2 (type of the Simon effect: egocentric Simon vs. allocentric Simon) $\times 2$ (type of the preceding spatial task: egocentric judgment vs. allocentric judgment) repeated-measures ANOVA. The results showed that neither the main effects of the type of Simon effect, $F_{(1,16)}=0.66, p=0.43$, nor the type of preceding spatial task, $F_{(1,16)}=2.88, p=0.11$, was significant. The two-way interaction, however, was significant, $F_{(1,16)}=$ 15.46, $p<0.01$. Further tests on the simple effects suggested that for the egocentric Simon effect, there was no significant difference between the two types of preceding spatial tasks, $t_{(16)}=0.64, p=0.53$. For the allocentric Simon effect, however, the effect size was significantly larger in the color tasks following the allocentric judgments $(156 \mathrm{~ms})$ than those following the egocentric judgments $(39 \mathrm{~ms}), t_{(16)}=$ $2.85, p=0.01$. These results suggested that the Simon effect based on the allocentric reference frame varied significantly depending on whether the allocentric representations were explicitly coded in the preceding blocks while the Simon effect based on the egocentric reference frame was free of such influences (Fig. 3). In addition, to test whether the Simon 


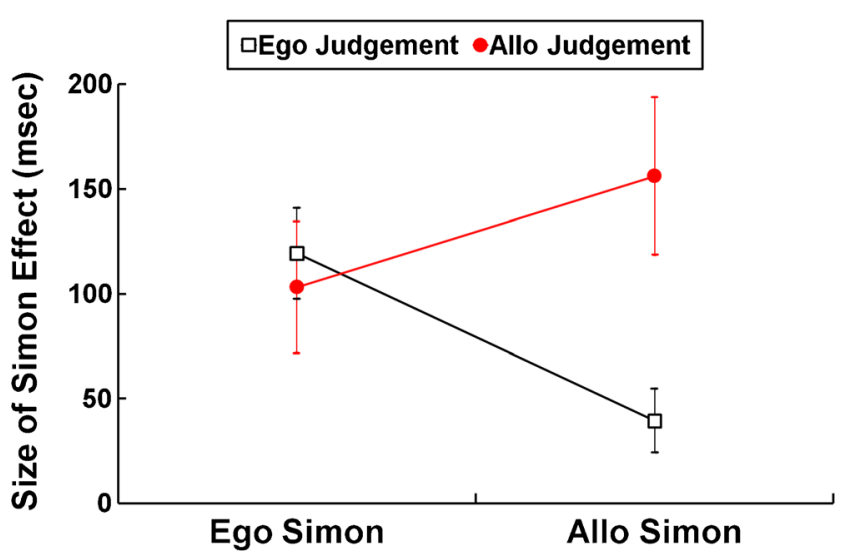

Fig. 3 The size of the allocentric versus the egocentric Simon effect in the color discrimination tasks changed as a function of the type of preceding spatial blocks (egocentric vs. allocentric) in Experiment 1. The error bars represent standard errors. The black line represents the sizes of the egocentric and allocentric Simon effects following the egocentric judgment tasks, respectively; the red line represents the sizes of the egocentric and allocentric Simon effects following the allocentric judgment tasks, respectively

effects were significant in all the four experimental conditions (i.e., the egocentric Simon effect following either egocentric or allocentric task, and the allocentric Simon effect following either egocentric or allocentric task), we performed planned $t$ tests on the RTs in the congruent and incongruent conditions in the four conditions. The results showed that there significant Simon effects did indeed exist in all four conditions despite the different sizes: the egocentric Simon effect following egocentric tasks $(119 \mathrm{~ms}), t_{(16)}=5.38, p<0.01$; the egocentric Simon effect following allocentric tasks $(103 \mathrm{~ms}), t_{(16)}=3.21$, $p<0.01$; the allocentric Simon effect following egocentric tasks (39 ms), $t_{(16)}=2.51, p=0.02$; and the allocentric Simon effect following allocentric tasks $(156 \mathrm{~ms}), t_{(16)}=$ $4.00, p<0.01$.

\section{Experiment 2}

In Experiment 1, both the allocentric and the egocentric representations were explicitly induced and coded based on the current task demands of allocentric and egocentric judgments. Moreover, since the non-spatial color discrimination task was interleaved with the allocentric and egocentric spatial judgment tasks, the explicitly induced allocentric and egocentric representations would interfere with the side of the response code and interacted with each other, causing the Simon effects based on both reference frames. It remained unknown, however, whether the allocentric and egocentric representations can be represented without explicit task demands, and cause interactive Simon effects based on both reference frames. Therefore, in Experiment 2, we asked a new group of participants to perform the color discrimination task without the interleaving spatial tasks.

\section{Method}

\section{Participants}

Twenty healthy participants (12 female and eight male, 1824 years old) took part in the present experiment. They were all right-handed, and had normal or corrected-to-normal vision as well as normal color vision. All the participants gave informed consent in accordance with the Declaration of Helsinki prior to the experiment and got paid for their performance afterwards.

\section{Apparatus, stimuli and experimental setup}

These were same as those in Experiment 1.

\section{Experimental design and tasks}

The only difference between the present experiment and Experiment 1 was that only the color discrimination task, rather than the spatial tasks, was performed in the present experiment.

\section{Results}

Incorrect responses and RTs outside three 3 SDs were excluded from further analysis. Mean RTs and error rates were then calculated for the four experimental conditions and were submitted to a 2 (the egocentric congruency: congruent vs. incongruent) $\times 2$ (the allocentric congruency: congruent vs. incongruent) repeated-measures ANOVA, respectively.

For RTs, the main effect of the egocentric congruency, $F_{(1,19)}=20.01, p<0.01$, suggested that the egocentric incongruent judgments $(546 \mathrm{~ms})$ were significantly slower than the egocentric congruent judgments $(516 \mathrm{~ms})$, i.e., a significant typical Simon effect based on the egocentric reference frame. The main effect of the allocentric congruency, however, was not significant, $F_{(1,19)}=0.02, p=0.90$, indicating that there was no significant Simon effect based on the allocentric reference frame (Fig. 4a). The two-way interaction was significant, $F_{(1,19)}=6.34, p<0.05$. Further tests on the simple effects were performed to clarify the interaction. For the egocentric congruent condition, no significant difference was found between the allocentric incongruent $(512 \mathrm{~ms})$ and congruent $(520 \mathrm{~ms})$ conditions, $t_{(19)}=1.38, p=0.18$. For the egocentric incongruent condition, there was also no significant difference between the allocentric incongruent $(549 \mathrm{~ms})$ and congruent (542 ms) conditions, $t_{(19)}=1.76, p=0.10$. For the allocentric congruent condition, RTs were significantly longer in the allocentric incongruent $(542 \mathrm{~ms})$ condition than in the allocentric congruent $(520 \mathrm{~ms})$ condition, $t_{(19)}=3.34$, $p<0.01$. For the allocentric incongruent condition, RTs were significantly longer in the allocentric incongruent 


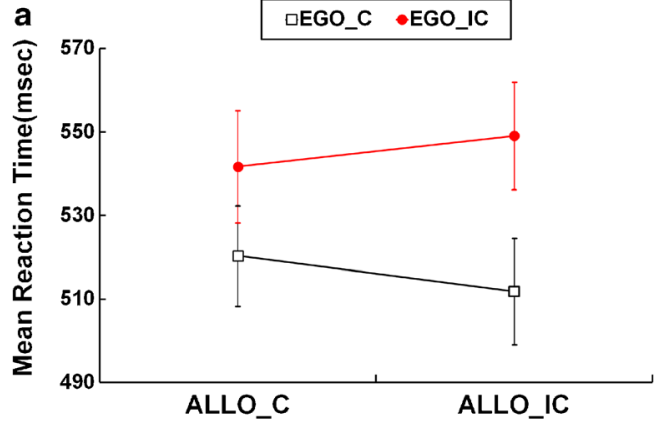

b

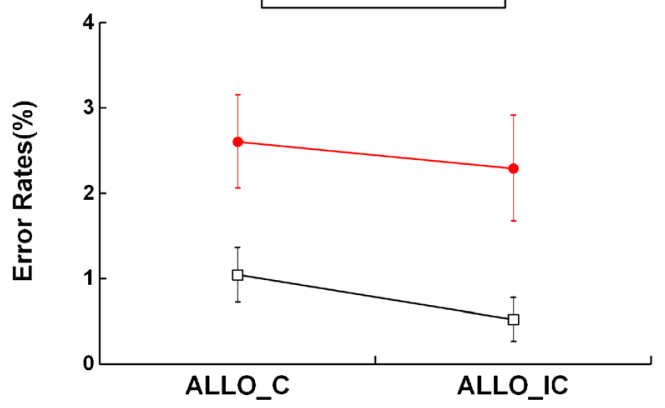

Fig. 4 Mean reaction times (a) and error rates (-) in the four experimental conditions in Experiment 2. The error bars represent standard errors

(549 ms) condition than in the allocentric congruent (512 ms) condition, $t_{(19)}=4.63, p<0.01$. Therefore, unlike Experiment 1 , RTs were not the longest when both allocentric and egocentric locations were incongruent with the response hand, and were not the shortest when both locations were congruent with the response hand. The pattern of the significant two-way interaction, however, was similar to that in Experiment 1. The size of the Simon effect based on the egocentric reference was significantly larger in the allocentric incongruent condition $(37 \mathrm{~ms})$ than in the allocentric congruent condition (21 ms). On the other hand, although the Simon effect based on the allocentric reference frame was not significant, its effect size was still affected by the egocentric congruency: the size of allocentric congruency was significantly larger in the egocentric incongruent condition $(7 \mathrm{~ms})$ than in the egocentric congruent condition ( $-8 \mathrm{~ms})$.

For the error rates, the only significant effect was the main effect of the egocentric congruency (Fig. $4 b), F_{(1,19)}=14.56$, $p<0.01$, indicating that participants made more errors in the egocentric incongruent trials $(2.5 \%)$ than in the egocentric congruent trials $(1.0 \%)$, irrespective of the allocentric congruency.

\section{Discussion}

By orthogonally crossing the Simon effect based on the egocentric and the allocentric positions in the present study, we aimed to answer the following three questions: (1) could the Simon effect be caused not only by the egocentric but also by the allocentric positions; (2) whether and how the Simon effect based on the egocentric and the allocentric reference frames interacted with each other; and (3) whether the potential interaction between them depended on task demands.

\section{The potential influence of affordance compatibility}

To improve the ecological effect of the study, the stimulus set adopted in the current study included two concrete but virtual objects (i.e., a fork on the top of a plate). Since the stimulus set included a graspable object (a fork), one may argue that the object affordance effect (Tucker \& Ellis, 1998) might be a confounding factor in the present results. The affordance effect refers to the phenomenon that viewing an object which relates to a particular action (e.g., a graspable object) can facilitate the response towards it when the response is compatible with the action evoked by the object, and hinder the response when incompatible (Tipper, Paul, \& Hayes, 2006; Tucker \& Ellis, 1998). Theoretically, affordances can be any motor plan brought about to utilize an object, such as orientation and grip aperture (e.g., Tucker \& Ellis, 1998, 2001). For example, previous studies on the affordance effect commonly used the orientation of an object as a key experimental manipulation, and revealed that participants' responses were faster when the orientation of the handle of a graspable object (a task-irrelevant property) corresponded with the side of the responding hand than when it did not (i.e., an orientation effect) (Pappas, 2014; Symes, Ellis, \& Tucker, 2005; Tucker \& Ellis, 1998). The term "orientation" here means the direction towards which the handle of a graspable object points (e.g., a hammer which oriented leftwards would have its handle pointing to the left side). Therefore, the critical factor, which results in the orientation effect, is the spatial compatibility between the orientation of a graspable object and the response hand. In the present study, however, the handle of the fork was always parallel to, rather than tilted to either side of, the mid-sagittal plane of the observers, and what varied was the position of the fork relative to either the plate or the observers' body. Therefore, due to the lack of spatial compatibility between the orientation of the handle of the fork and the response hand, we believe that the affordance effect would not be a major confounding factor in the present study.

In one of the previous studies, to dissociate the affordance effect and the Simon effect, the orientation and the egocentric position of a graspable target were simultaneously manipulated, and participants were asked to perform a color discrimination task (Symes et al., 2005). Their results showed that only the Simon effect existed in the color discrimination tasks, whereas the affordance effect was not observed. In the control experiment (Experiment 2) of the present study, in which only the color discrimination task, but not the spatial judgment 
tasks, was performed, we also observed only the Simon effect based on the egocentric positions, but not the Simon effect based on the allocentric positions. Thus, one might argue that our results were similar to those of Symes et al. (2005). However, we would like to point out that despite the seemingly similar results in the color discrimination tasks of the two studies, there existed essential differences between the present study and Symes et al. (2005).

First, although the egocentric reference frame adopted by the participants was the same between the two studies, the spatial reference frame, which was adopted by the affordance effect in Symes et al. (2005) and by the allocentric Simon effect in the present study was different. Specifically, although both are object-based, the orientation of the handle of an object in Symes et al. (2005) and the spatial position of an object relative to another object in the present study are essentially different. The discrimination of object's orientation would activate the dorsal visual stream in the parietal lobe, which is associated with visually guided actions (Grèzes, Tucker, Armony, Ellis, \& Passingham, 2003; Handy, Grafton, Shroff, Ketay, \& Gazzaniga, 2003; Riddoch et al., 2004). However, the allocentric representations are encoded in the ventral visual stream, which subserves visual awareness of object properties (Chen, Weidner, Weiss, Marshall, \& Fink, 2012; Georges-François, Rolls, \& Robertson, 1999; James, Culham, Humphrey, Milner, \& Goodale, 2003; James, Humphrey, Gati, Menon, \& Goodale, 2002; Rolls, 1999). Second, the pattern of interaction was different between the two experiments. In our control experiment, although the allocentric Simon effect was not observed, the interaction between the egocentric and allocentric Simon effect still existed, which was similar to that in the main experiment. However, in Experiment 3 (color task) of Symes et al. (2005), neither the affordance effect nor the interaction between the object orientation and the object position was obtained, again indicating that the affordance effect and the allocentric Simon effect are two different phenomena.

\section{The Simon effect based on egocentric and allocentric reference frames}

In Experiment 1, to make sure that the allocentric and egocentric positions of the targets were explicitly induced and represented, the non-spatial color discrimination task was interleaved with the allocentric and egocentric spatial judgment tasks. The results suggested that there were significant Simon effects based on not only the egocentric but also the allocentric reference frame (Fig. 2a). Further analysis on how the size of the two types of Simon effects (egocentric vs. allocentric) changed as a function of the type of preceding spatial judgment task (egocentric vs. allocentric) showed that although there were significant egocentric and allocentric Simon effects irrespective of the type of the preceding spatial task, the type of the preceding spatial task could indeed affect the size of the Simon effect (Fig. 3). Specifically, although the size of the egocentric Simon effect did not change as a function of the preceding spatial task, the size of the allocentric Simon effect was significantly larger when the preceding spatial task was allocentric than egocentric task (Fig. 3).

Since the allocentric and egocentric representations were explicitly induced by the interleaving spatial tasks in Experiment 1, it remained unclear whether the Simon effect based on the egocentric and the allocentric reference frames existed and interacted upon the appearance of the target without explicit demands of spatial judgments. To further investigate this issue, a new group of participants were asked to perform only the non-spatial color discrimination task, but not the interleaving spatial judgment tasks, in Experiment 2. The results suggested that without explicit demands on coding the egocentric or the allocentric representations, there was a significant Simon effect based on only the egocentric, rather than the allocentric, reference frame (Fig. 4). Therefore, together with the results in Experiment 1, our results suggested that the egocentric Simon effect existed irrespective of whether the egocentric positions were explicitly induced by the current task demands or not, whereas the allocentric Simon effect seemed to occur only when the allocentric positions were explicitly induced.

The classic Simon effect refers to the phenomenon that responses are faster when the task-irrelevant egocentric stimulus location is on the same side as the response than when it is not (Lu \& Proctor, 1995; Simon, Hinrichs, \& Craft, 1970; Simon \& Small, 1969; Zhang, Zhou, di Pellegrino, \& Ladavas, 2007). Even when the egocentric stimulus location is irrelevant, a spatial representation of the egocentric location is still coded, together with a spatial code of the response to the relevant stimulus dimension (e.g., color or shape). Responses are slower when the two spatial codes are incongruent than when they are congruent. Although the spatial code of the typical Simon effect is defined with regard to the egocentric reference frame, the spatial location of external stimuli often varies according to which reference frame the stimulus location is represented in. Our results suggested that the egocentric Simon effect existed irrespective of whether the egocentric representations were explicitly coded or not, while the allocentric Simon effect appeared to exist only when the allocentric representations were explicitly induced by the current task demands. Upon first encountering an environment, people immediately form multiple representations of space (Graziano, Yap, \& Gross, 1994; Mou, McNamara, Valiquette, \& Rump, 2004; Tipper \& Behrmann, 1996; Tversky, Lee, \& Mainwaring, 1999). Previous evidence further showed that in socialization situations, such as communicating with others and simple social action, people spontaneously adopt another's perspective rather than their own, 
while in the common natural situation the egocentric perspective on space has primacy (Epley et al., 2004; Piaget \& Inhelder, 1956; Tversky \& Hard, 2009). Consistent with the previous evidence, in the natural non-social situation of the present study, the egocentric positions were automatically coded and interacted with the responding hand independent of task demands, whereas the allocentric positions seemed to be explicitly coded and interacted with the responding hand only when needed.

However, although the existence of the allocentric Simon effect appeared to depend on whether the allocentric positions were explicitly coded as required by the current task demands, the allocentric representations still seemed to either explicitly (Experiment 1) or implicitly (Experiment 2) interact with the egocentric Simon effect in a similar way since similar patterns of interaction between the egocentric and allocentric Simon effect were observed in Experiments 1 and 2 (Figs. 2a and 4a).

\section{Interaction between the egocentric and the allocentric Simon effect}

In the present study, the egocentric and allocentric Simon effect showed similar patterns of interaction in Experiments 1 and 2, irrespective of whether the spatial locations of the target were explicitly coded or not (Figs. 2a and 4a). The Simon effect based on one reference frame was significantly enhanced when the target position with regard to the other reference frame was incongruent with the response code compared to when it was congruent. The significant pattern of interaction was driven by the significantly delayed responses when the target position was incongruent with the response code in both the egocentric and the allocentric reference frames (Figs. 2a and 4a).

This pattern of interaction could be explained by the dimensional overlap model (Kornblum et al., 1990). Dimensional overlap is defined as a similarity of perceptual, conceptual, or structural attributes between any combinations of: (1) the relevant dimension of the stimulus set and the response set, (2) the irrelevant dimension of the stimulus set and the response set, and (3) the relevant and irrelevant dimensions of the stimulus set. The factorial combination of these three factors results in eight types of potential SR ensembles (Kornblum et al., 1990; Kornblum \& Lee, 1995; Kornblum, Stevens, Whipple, \& Requin, 1999; Liu, Park, Gu, \& Fan, 2010; Zhang \& Kornblum, 1998). According to this model, there is dimensional overlap between the irrelevant stimulus dimension $\left(\mathrm{S}_{\mathrm{I}}\right.$; e.g., left of mid-sagittal plane or left side of plate) and the response dimension (R; e.g., press left-hand key), whereas no overlap exists between the relevant stimulus dimension $\left(\mathrm{S}_{\mathrm{R}}\right.$; e.g., dark gray) and $\mathrm{S}_{\mathrm{I}}$ or between $\mathrm{S}_{\mathrm{R}}$ and $\mathrm{R}$ in a typical Simon task (Kornblum et al., 1990; Kornblum \& Lee, 1995; Kornblum et al., 1999; Zhang \&
Kornblum, 1998). The dimensional overlap between $S_{R}, S_{I}$, and $\mathrm{R}$ determines the $\mathrm{S}-\mathrm{R}$ incompatibility. If the irrelevant stimulus and the response sets have overlapping dimensions, the presentation of the irrelevant stimulus set automatically activates its corresponding response location. When the irrelevant stimulus dimension is the same as the response location, the response will be speeded up but slowed down when the two conflict (Kornblum, 1994; Kornblum et al., 1990; Lu \& Proctor, 1995). In our experiment, there were two types of $\mathrm{S}_{\mathrm{I}}$ dimensions in the color discrimination task: the egocentric and allocentric locations of the target. According to the dimensional overlap model, the interference effect will be larger if the two pairs of dimensions both conflict than if only one pair does, while the facilitation effect will be stronger if the two pairs of dimensions are both congruent than if only one pair is congruent (Liu et al., 2010). The results of Experiment 1 indeed showed that RTs were significantly shorter when the two $\mathrm{S}_{\mathrm{I}}$ dimensions were both congruent with the response code, and were significantly longer when the two $\mathrm{S}_{\mathrm{I}}$ dimensions were both incongruent with the response code (Fig. 2a). In Experiment 2, however, RTs were not the longest when both types of locations were incongruent with the response hand and were not the shortest when both types of locations were congruent with the response hand. One possible account for this discrepancy between Experiments 1 and 2 could be that dimensional overlap affects task performance only when all dimensions that give rise to the facilitation or the interference effect are explicitly coded as required by the current task demands. Since one $\mathrm{S}_{\mathrm{I}}$ dimension (i.e., the allocentric position) in the present Experiment 2 seemed to be implicitly coded, dimensional overlap did not work as effectively as that in Experiment 1.

To summarize, by orthogonally crossing the SR compatibility with regard to the egocentric and allocentric reference frames in the present study, we revealed that the classical Simon effect based on the egocentric reference frame existed irrespective of whether the egocentric positions were explicitly coded or not, while the Simon effect based on the allocentric reference frame seemed to occur only when the allocentric positions were induced by the current task demands. Moreover, the egocentric and allocentric Simon effect interacted in a similar way independent of task demands with significantly delayed responses when both the egocentric and the allocentric positions were incongruent with the response code.

Acknowledgments HW and NL contributed equally to the present study. The work was supported by grants from the Natural Science Foundation of China (31371127). QC is supported by the Program for New Century Excellent Talents in the University of China (NCET-120645) and by the Guangdong Province Universities and Colleges Pearl River Scholar Funded Scheme (2014). HW is supported by the Scientific Research Foundation of Graduate School of South China Normal University (2015). 


\section{References}

Bisiach, E., Capitani, E., \& Porta, E. (1985). Two basic properties of space representation in the brain: Evidence from unilateral neglect. Journal of Neurology, Neurosurgery \& Psychiatry, 48(2), 141-144.

Chen, Q., Weidner, R., Weiss, P. H., Marshall, J. C., \& Fink, G. R. (2012). Neural interaction between spatial domain and spatial reference frame in parietal-occipital junction. Journal of Cognitive Neuroscience, 24(11), 2223-2236.

Driver, J., \& Halligan, P. W. (1991). Can visual neglect operate in objectcentred co-ordinates? An affirmative single-case study. Cognitive Neuropsychology, 8(6), 475-496.

Epley, N., Morewedge, C. K., \& Keysar, B. (2004). Perspective taking in children and adults: Equivalent egocentrism but differential correction. Journal of Experimental Social Psychology, 40(6), 760-768.

Fitts, P. M., \& Deininger, R. L. (1954). SR compatibility: Correspondence among paired elements within stimulus and response codes. Journal of Experimental Psychology, 48(6), 483.

Fitts, P. M., \& Seeger, C. M. (1953). SR compatibility: Spatial characteristics of stimulus and response codes. Journal of Experimental Psychology, 46(3), 199.

Georges-François, P., Rolls, E. T., \& Robertson, R. G. (1999). Spatial view cells in the primate hippocampus: Allocentric view not head direction or eye position or place. Cerebral Cortex, 9(3), 197-212.

Graziano, M. S. A., Yap, G. S., \& Gross, C. G. (1994). Coding of visual space by premotor neurons. Science, 266(5187), 1054-1057.

Grèzes, J., Tucker, M., Armony, J., Ellis, R., \& Passingham, R. E. (2003). Objects automatically potentiate action: An fMRI study of implicit processing. European Journal of Neuroscience, 17(12), 2735-2740.

Handy, T. C., Grafton, S. T., Shroff, N. M., Ketay, S., \& Gazzaniga, M. S. (2003). Graspable objects grab attention when the potential for action is recognized. Nature Neuroscience, 6(4), 421-427.

Hedge, A., \& Marsh, N. W. A. (1975). The effect of irrelevant spatial correspondences on two-choice response-time. Acta Psychologica, 39(6), 427-439.

Hommel, B. (1994). Spontaneous decay of response-code activation. Psychological Research, 56(4), 261-268.

James, T. W., Culham, J., Humphrey, G. K., Milner, A. D., \& Goodale, M. A. (2003). Ventral occipital lesions impair object recognition but not object-directed grasping: An fMRI study. Brain: A Journal of Neurology, 126(11), 2463-2475(2413).

James, T. W., Humphrey, G. K., Gati, J. S., Menon, R. S., \& Goodale, M. A. (2002). Differential effects of viewpoint on object-driven activation in dorsal and ventral streams. Neuron, 35(4), 793-801.

Kornblum, S. (1994). The way irrelevant dimensions are processed depends on what they overlap with: The case of Stroop-and Simon-like stimuli. Psychological Research, 56(3), 130-135.

Kornblum, S., Hasbroucq, T., \& Osman, A. (1990). Dimensional overlap: cognitive basis for stimulus-response compatibility: A model and taxonomy. Psychological Review, 97(2), 253-270.

Kornblum, S., \& Lee, J.-W. (1995). Stimulus-response compatibility with relevant and irrelevant stimulus dimensions that do and do not overlap with the response. Journal of Experimental Psychology: Human Perception and Performance, 21(4), 855-875.

Kornblum, S., Stevens, G. T., Whipple, A., \& Requin, J. (1999). The effects of irrelevant stimuli: 1 . The time course of stimulus-stimulus and stimulus-response consistency effects with Stroop-like stimuli, Simon-like tasks, and their factorial combinations. Journal of Experimental Psychology: Human Perception and Performance, 25(3), 688-714.

Lamberts, K., Tavernier, G., \& d'Ydewalle, G. (1992). Effects of multiple reference points in spatial stimulus-response compatibility. Acta Psychologica, 79(2), 115-130.
Liu, X., Park, Y., Gu, X., \& Fan, J. (2010). Dimensional overlap accounts for independence and integration of stimulus - response compatibility effects. Attention, Perception, \& Psychophysics, 72(6), 1710-1720.

Lu, C., \& Proctor, R. W. (1995). The influence of irrelevant location information on performance: A review of the Simon and spatial Stroop effects. Psychonomic Bulletin \& Review, 2(2), 174-207.

Marble, J. G., \& Proctor, R. W. (2000). Mixing location-relevant and location-irrelevant choice-reaction tasks: Influences of location mapping on the Simon effect. Journal of Experimental Psychology: Human Perception and Performance, 26(5), 1515.

Marshall, J. C., \& Halligan, P. W. (1993). Visuo-spatial neglect: A new copying test to assess perceptual parsing. Journal of Neurology, 240(1), 37-40

Mou, W., McNamara, T. P., Valiquette, C. M., \& Rump, B. (2004). Allocentric and egocentric updating of spatial memories. Journal of Experimental Psychology: Learning, Memory, and Cognition, $30(1), 142-157$.

Pappas, Z. (2014). Dissociating Simon and affordance compatibility effects: Silhouettes and photographs. Cognition, 133(3), 716-728.

Piaget, J., \& Inhelder, B. (1956). The child's concept of space. New York: Humanities Pr.

Proctor, R. W., \& Pick, D. F. (2003). Display-control arrangement correspondence and logical recoding in the Hedge and Marsh reversal of the Simon effect. Acta Psychologica, 112(3), 259-278.

Riddoch, M. J., Humphreys, G. W., Jacobson, S., Pluck, G., Bateman, A., \& Edwards, M. (2004). Impaired orientation discrimination and localisation following parietal damage: On the interplay between dorsal and ventral processes in visual perception. Cognitive Neuropsychology, 21(6), 597-623.

Rolls, E. T. (1999). Spatial view cells and the representation of place in the primate hippocampus. Hippocampus, 9(4), 467-480.

Simon, J. R. (1990). The effects of an irrelevant directional cue on human information processing. Advances in Psychology, 65, 31-86.

Simon, J. R., Hinrichs, J. V., \& Craft, J. L. (1970). Auditory SR compatibility: Reaction time as a function of ear-hand correspondence and ear-response-location correspondence. Journal of Experimental Psychology, 86(1), 97-102.

Simon, J. R., \& Small, A. M., Jr. (1969). Processing auditory information: Interference from an irrelevant cue. Journal of Applied Psychology, 53(5), 433-435.

Symes, E., Ellis, R., \& Tucker, M. (2005). Dissociating object-based and space-based affordances. Visual Cognition, 12(7), 1337-1361.

Tipper, S. P., \& Behrmann, M. (1996). Object-centered not scene-based visual neglect. Journal of Experimental Psychology: Human Perception and Performance, 22(5), 1261-1278.

Tipper, S. P., Paul, M. A., \& Hayes, A. E. (2006). Vision-for-action: The effects of object property discrimination and action state on affordance compatibility effects. Psychonomic Bulletin \& Review, 13(3), 493-498.

Tucker, M., \& Ellis, R. (1998). On the relations between seen objects and components of potential actions. Journal of Experimental Psychology: Human Perception and Performance, 24(3), 830.

Tucker, M., \& Ellis, R. (2001). The potentiation of grasp types during visual object categorization. Visual Cognition, 8(6), 769-800.

Tversky, B., \& Hard, B. M. (2009). Embodied and disembodied cognition: Spatial perspective-taking. Cognition, 110(1), 124-129.

Tversky, B., Lee, P., \& Mainwaring, S. (1999). Why do speakers mix perspectives? Spatial Cognition and Computation, 1(4), 399-412.

Umiltà, C., \& Liotti, M. (1987). Egocentric and relative spatial codes in SR compatibility. Psychological Research, 49(2-3), 81-90.

Vallesi, A., Mapelli, D., Schiff, S., Amodio, P., \& Umilta, C. (2005). Horizontal and vertical Simon effect: Different underlying mechanisms? Cognition, 96(1), B33-B43.

Walker, R. (1995). Spatial and object-based neglect. Neurocase, 1(4), 371-383. 
Wühr, P. (2006). The Simon effect in vocal responses. Acta Psychologica, 121(2), 210-226.

Zhang, H., \& Kornblum, S. (1998). The effects of stimulus-response mapping and irrelevant stimulus-response and stimulus-stimulus overlap in four-choice Stroop tasks with single-carrier stimuli.
Journal of Experimental Psychology: Human Perception and Performance, 24(1), 3-19.

Zhang, D., Zhou, X., di Pellegrino, G., \& Ladavas, E. (2007). Spatial coding for the Simon effect in visual search. Experimental Brain Research, 176(4), 616-629. 